\title{
Comparison of Computer and Human Face Recognition According to Facial Components
}

\author{
Hyun Ha Nam ${ }^{\dagger}$, Byung Jun Kang ${ }^{+\dagger}$, Kang Ryoung Park ${ }^{+++}$
}

\section{ABSTRACT}

Face recognition is a biometric technology used to identify individuals based on facial feature information. Previous studies of face recognition used features including the eye, mouth and nose; how ${ }^{-}$ ever, there have been few studies on the effects of using other facial components, such as the eyebrows and chin, on recognition performance. We measured the recognition accuracy affected by these facial components, and compared the differences between computer-based and human-based facial recognition methods. This research is novel in the following four ways compared to previous works. First, we meas ${ }^{-}$ ured the effect of components such as the eyebrows and chin. And the accuracy of computer-based face recognition was compared to human-based face recognition according to facial components. Second, for computer-based recognition, facial components were automatically detected using the Adaboost algorithm and active appearance model (AAM), and user authentication was achieved with the face recognition algorithm based on principal component analysis (PCA). Third, we experimentally proved that the number of facial features (when including eyebrows, eye, nose, mouth, and chin) had a greater impact on the accuracy of human-based face recognition, but consistent inclusion of some feature such as chin area had more influence on the accuracy of computer-based face recognition because a computer uses the pixel values of facial images in classifying faces. Fourth, we experimentally proved that the eyebrow feature enhanced the accuracy of computer-based face recognition. However, the problem of occlusion by hair should be solved in order to use the eyebrow feature for face recognition.

Key words: Computer-based face recognition, human-based face recognition, facial components

\section{INTRODUCTION}

Face recognition is a biometric technology for user authentication using facial features. A computer-based face recognition system finds the correspondence between enrolled and input facial

※ Corresponding Author: Kang Ryoung Park, Address : Division of Electronics and Electrical Engineering, Dongguk University, 26, Pil-dong 3-ga, Chung-gu, Seoul, Republic of Korea (100-715), TEL : +82-10-3111-7022, FAX : +82-2-2277-8735, E-mail : parkgr@dgu.edu

Receipt date : Sep. 19, 2011, Revision date : Oct. 28, 2011 Approval date: Nov. 22, 2011

${ }^{+}$Department of Computer Science, Graduate School of Information Science and Engineering, Tokyo Institute of Technology

(E-mail: hyunha@sg.cs.titech.ac.jp)

${ }^{++}$Technical Research Institute, Hyundai Mobis (E-mail: kangbyj@mobis.co.kr)

${ }^{+++}$Division of Electronics and Electrical Engineering, Dongguk University images. Such systems are used for door control, immigration administration, and user authentication for mobile devices because they can be easily implemented while not requiring a user to touch the device.

Previous studies of face recognition have used the eyes, mouth, and nose; however, there has been little research on the effect of other facial components such as eyebrows and the chin on recognition performance [1-3]. Sadrô et al. suggested that the absence of eyebrows leads to significant confusion in the perception process [4]. However, they did not discuss the influence of other facial components, and only focused on human-based recognition without considering computer-based recognition. O'Toole et al. showed a comparison between computer-based and human-based face recognition according to illumination variation [5]. 
Verma et al. presented a novel methodology for quantitative analysis of changes in facial display as the intensity of an emotion evolves from neutral to peak expression based on regional volumetric difference (RVD) [6]. Gur et al. described a method for accurately acquiring and reconstructing the geometry of the human face and for display of this reconstruction in a 3-dimensional format. They applied the method in a sample of 70 actors and 69 actresses expressing happiness, sadness, anger, fear and disgust, as well as neutral expressions [7]. Maeng et al. proposed the method of evaluating face recognition system based on scenarios [8]. However, there is no research that considers the difference between computer-based and human-based face recognition according to facial components.

The numerous methods of computer-based face recognition [9] are divided into appearance-based and feature-based face approaches. Appearancebased methods include principal component analysis (PCA), linear discriminant analysis (LDA), and independent component analysis (ICA); these methods reconstruct a face image in a subspace consisting of a set of basis vectors. A PCA-based method is an appearance-based face recognition algorithm and expresses a face image as linear combination of eigen-faces [10-14]. An LDAbased method determines class classifications using Fisher vectors. In general, LDA is reported to show better performance for cases with variations in illumination and expression, but is more sensitive to image alignment than the PCA-based methods. In addition, the conventional LDA method has the disadvantage that the separability criteria are not directly related to the classification ability of the obtained component representation. To solve this problem, direct LDA (D-LDA) [15,16], general discriminant analysis (GDA) [17], and kernel Fisher discriminant analysis (KFDA) were developed [18-22]. An ICA-based method expresses a facial image with statistically-independent compo- nents. In this approach, the independent components must have non-Gaussian distributions and the unknown mixing matrix is square [23].

In contrast, feature-based face recognition uses angles, distance, and the position of geometric facial component points [24-28]. The elastic bunch graph-matching (EBGM) approach performs the recognition process using graph models based on the relative positions of facial components [28] However, the performance of feature-based face recognition is very sensitive to image alignment and the detection accuracy of facial component points. So, we used the PCA-based face recognition algorithm as a computer-based one because it has been widely used due to its robustness under the environment without illumination and $\mathrm{ex}^{-}$ pression variation.

The structure of this paper is as follows. In Section 2, we introduce computer-based face recognition which uses Adaboost, AAM, and PCA, and a human-based face recognition method. Experimental results are described in Section 3, and conclusions are provided in Section 4.

\section{THE PROPOSED METHOD}

\subsection{Computer-based face recognition}

\subsubsection{Overview}

Fig. 1 shows the overall procedure for computer-based face recognition. An input image captured by a camera contains the background regions as well as a human face, so the face region must be isolated before extracting facial features. To measure recognition accuracy according to the facial components, the active appearance model (AAM) was used for detecting each facial component [29-31]. Since its performance is sensitive to the initial position of the face region, we precisely determined the initial position by using adaptive boosting (Adaboost) face and eye detectors before applying the AAM algorithm. The Adaboost meth- 


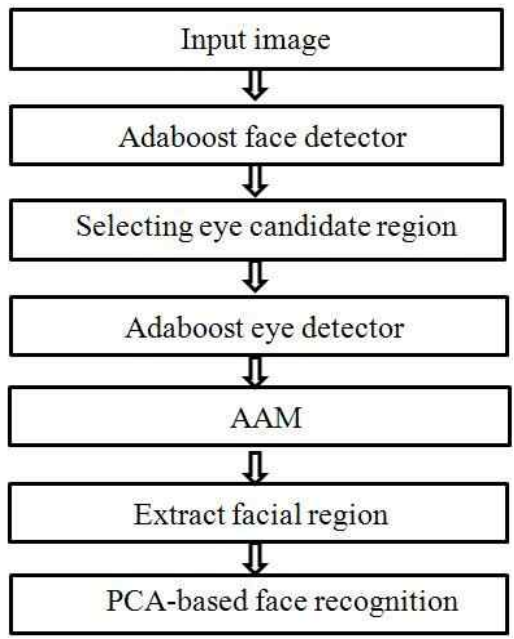

Fig 1. Overall procedure of the computer-based face recognition.

od is a machine learning algorithm and generally constructs a strong classifier by combining several weak classifiers based on Haar-like features [32,33]. This algorithm takes a long time to train weak classifiers, but is very fast in testing. After detecting the face region with the Adaboost face detector, two eye searching regions were defined from the detected face region. Two eye positions were also detected by using the Adaboost eye de $^{-}$ tector in the eye searching regions, and were then used for the initial positions of the AAM, as shown in Fig. 2(a)

As shown in Fig. 2(b), the AAM determined fa-

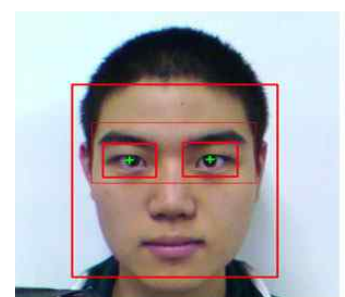

(a)

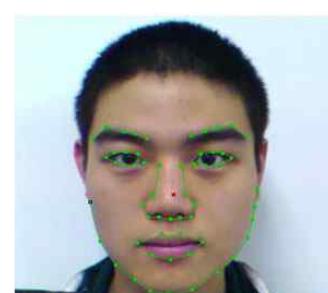

(b)
Fig. 2. Detection of face, eyes and facial feature points. (a) Face and eye detection using the Adaboost algorithm (largest red box represents the facial region). (b) Extraction of facial feature points using AAM (red point represents the geometric center of all the detected facial feature points). cial components using the shape and appearance information of the face. Because the Adaboost face detector can give a rough facial box as shown in Fig. 2(a), an accurate facial region should be $\mathrm{de}^{-}$ tected by the AAM in order to measure the accuracy of facial recognition according to facial components.

From the accurate AAM face region, facial features such as eigen-coefficients by PCA were extracted and authentication was performed. If the Euclidean distance between the enrolled $\mathrm{CO}^{-}$ efficients and those of the input image is less than a predetermined threshold, the input face is accepted as an enrolled user. If the distance is greater than the threshold, the input image is rejected as an un-enrolled user.

\subsubsection{Facial feature extraction based on AAM}

The AAM is non-linear, generative, and parametric model of a visual phenomenon. It is composed of a statistical shape and statistical appearance models represented by a linear parametric model such as PCA [34-36]. The shape model is represented by a linear combination of a mean shape vector $\mathrm{S}_{0}$ and $\mathrm{m}$ shape basis vectors $\mathrm{S}_{i}$ which are composed of feature points in two dimensional $(2-\mathrm{D})$ coordinates such that $\mathrm{S}_{\mathrm{i}}=\left(x_{1}, y_{1}\right.$, $\left.\cdots, x_{l}, y_{l}\right)^{\mathrm{T}}$.

$$
\mathbf{S}=\mathbf{S}_{0}+\sum_{i=1}^{m} p_{i} \mathbf{S}_{i}
$$

where the coefficient $p_{i}$ denotes the $I^{\text {th }}$ shape parameter. Here, the shape basis vectors $\mathrm{S}_{i}$ are assumed to be orthonormal [35]. The shape model S is trained with feature points which are manually marked in the training data set. The mean shape $\mathrm{S}_{0}$ and the shape basis vectors $\mathrm{S}_{i}$ are obtained by PCA. As shown in Fig. 2(b), we used 58 feature points for the shape model. The training images were co-registered with the mean shape using a piecewise affine method, and the appearance model was trained from the aligned training images. The 
appearance model is represented by a linear combination of a mean appearance vector $\mathrm{A}_{0}(\mathrm{x})$ and $\mathrm{n}$ appearance basis vectors $\mathrm{A}_{i}(\mathrm{x})$ [34-36]:

$$
\mathrm{A}(\mathrm{x})=\mathrm{A}_{0}(\mathrm{x})+\sum_{i=1}^{n} \alpha_{i} \mathrm{~A}_{i}(\mathrm{x}) \quad \forall \mathrm{x} \in \mathbf{p}\left(\mathrm{S}_{0}\right)
$$

where $\mathrm{p}\left(\mathrm{S}_{0}\right)$ is the set of pixels $\mathrm{x}=(\mathrm{x}, \mathrm{y})^{\mathrm{T}}$ which lay inside the mean facial shape $\mathrm{S}_{0}, \mathrm{~A}_{0}(\mathrm{x})$ is the intensity value of the facial appearance at point $\mathrm{x}$, and the coefficient $a_{i}$ denotes the $i^{\text {th }}$ appearance parameter. The image $\mathrm{A}_{i}(\mathrm{x})$ is assumed to be orthonormal. The mean appearance $\mathrm{A}_{0}(\mathrm{x})$ and $n$ appearance basis vectors are generally gained by applying PCA to the training data set [34-36].

$$
E=\sum_{\mathbf{x} \in \mathbf{p}\left(s_{n}\right)}\left[\mathrm{A}_{0}(\mathbf{x})+\sum_{i=1}^{m} \alpha_{i} \mathrm{~A}_{i}(\mathbf{x})-\mathrm{I}(\mathrm{W}(\mathbf{x} ; \mathbf{p}))\right]^{2}
$$

where $\mathrm{W}(\mathrm{x} ; \mathrm{p})$ denotes a piecewise affine warp performed over all pixels $\mathrm{x}$ in the base mesh $\mathrm{S}_{0}$. The AAM was trained by minimizing the $E$ value in Eq. (3) [35]. After training the AAM, we automatically obtained the coordinates of the facial feature points through AAM fitting.

The initial position is required to acquire the $\mathrm{fa}^{-}$ cial feature points in an input image using AAM fitting. Because the performance of the AAM is very sensitive to initial position, accurate selection of the initial position is very important. Therefore, we accurately determined the initial position by $\mathrm{de}^{-}$ tecting two eye positions. They were detected using the Adaboost eye detector in the eye searching region defined in the detected face area using the Adaboost face detector, as shown in Figs. 1 and 2(a).

\subsection{3 $\mathrm{PCA}$-based face recognition}

PCA is a criterion technique to approximate original data with lower dimensional feature vectors $[10,37,38]$. Let the training set of face images be $\mathrm{I}_{1}, \mathrm{I}_{2}, \cdots \mathrm{I}_{\mathrm{M}}$ and then the mean face of the set is defined by

$$
\mathrm{O}=\frac{1}{M} \sum_{i=1}^{M} \mathrm{I}_{i}
$$

The difference between each face and the mean face can be represented by the vectors $\Phi_{i}=I_{i}-O$. The covariance matrix $C$ is expressed by eigen-values and eigen-vectors as follows:

$$
\mathrm{C}=\frac{1}{M} \sum_{i=1}^{M} \Phi_{i} \cdot \Phi_{i}^{\mathrm{T}}
$$

The eigen-vectors $u_{i}$ and eigen-values $\lambda_{i}$ are obtained as follows:

$$
\mathrm{C} u_{i}=\lambda_{i} u_{i}
$$

Let a testing image be $\mathrm{I}_{\text {test }}$. They are projected into subspace by following expression:

$$
\omega_{\text {test }}=\mathrm{U}^{\mathrm{T}}\left(\mathrm{I}_{\text {test }}-\mathrm{O}\right)
$$

where $\mathrm{U}$ denotes the matrix which consists of eigen-vectors $u_{i}$. In addition, the weight vector $\omega_{\mathrm{k}}$ describes the contribution of each eigen-vector in the test image, and is named as an eigen- ${ }^{-}{ }^{-}$ efficient vector. PCA-based face recognition $\mathrm{de}^{-}$ termines the result of user authentication by calculating the Euclidean distance between the weighted vectors of the enrolled image $\left(\omega_{\mathrm{E}}\right)$ and the input image $\left(\omega_{\mathrm{R}}\right)$ as follows [39]:

$$
E D=\left\|\omega_{E}-\omega_{R}\right\|
$$

If the Euclidean distance between the $\mathrm{CO}^{-}$ efficients of an enrolled image and an input one is less than a predetermined threshold, the input face is accepted as an enrolled user. If it is greater than the threshold, the input image is rejected as an un-enrolled user. We experimented with the training image of our database in order to obtain the smallest equal error rate (EER) of face recognition according to various thresholds. From that, the optimal threshold was empirically determined.

\subsubsection{Stimuli}

As shown in Fig. 3, we classified four cases 

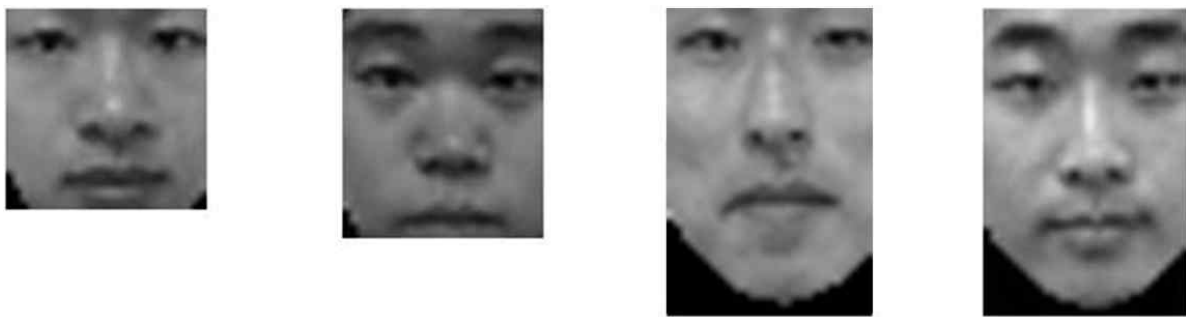

Fig. 3. Four cases of facial image according to facial components.

based on the facial feature points detected by the AAM in order to measure recognition accuracy according to facial components. The facial image of the first case consisted of the eyes, nose, and mouth, which were mainly used for conventional face recognition. The second and third cases added the eyebrows and chin to the first case, $\mathrm{re}^{-}$ spectively. The fourth case consisted of facial images which added the eyebrows and chin to the first case. In each case, the background was $\mathrm{ex}^{-}$ cluded by the zero padding method after shifting the mean level. Here, the shifting of the mean level corresponded to the subtraction by $\mathrm{O}$ as shown in Eq. (7). We made the pixel of background as 0 for minimizing the effect of background as shown in Fig. 3, the calculated eigen-coefficient values in the background area using Eq. (7) also become 0, and thus did not affect the calculation of dissimilarity between the enrolled coefficients and those from the input image.

\subsubsection{Procedure}

The computer-based face recognition procedure is as follows:

(1) PCA eigen-vectors (U of Eq. (7)) and AAM models ( $\mathrm{S}_{0}$ and $\mathrm{S}_{\mathrm{i}}$ of Eq.(1) and $\mathrm{Ad}(\mathrm{x})$ and $\mathrm{A}_{i}(\mathrm{x})$ of Eq. (2)) were constructed from the training set according to the four cases shown in Fig. 3.

(2) The recognition process was performed as shown in Fig. 1. The optimal number of eigenvectors was empirically determined such that the smallest EER of face recognition was obtained.

(3) The accuracy of computer-based face recognition was measured for each case in Table 1.

\subsection{Human-based face recognition}

\subsubsection{Participants}

Twenty subjects participated in the experiment for human-based face recognition. The volunteers consist of two female and eighteen male university students.

\subsubsection{Stimuli}

To accomplish fair experiments, 480 images used for computer-based face recognition were also used for human-based recognition. In addition, the face images of 20 participants were not included in the experiment in order to prevent them from grasping their face images, easily. The participants evaluated the face images of each case

Table 1. Four cases of face images according to facial components

\begin{tabular}{|c|c|c|c|c|}
\hline & $\begin{array}{c}\text { Case 1 } \\
\text { (Fig. 3 (a)) }\end{array}$ & $\begin{array}{c}\text { Case 2 } \\
\text { (Fig. 3 (b)) }\end{array}$ & $\begin{array}{c}\text { Case 3 } \\
(\text { Fig. 3 (c)) }\end{array}$ & $\begin{array}{c}\text { Case 4 } \\
\text { (Fig. 3 (d)) }\end{array}$ \\
\hline \hline $\begin{array}{c}\text { Included } \\
\text { facial components }\end{array}$ & Eye, Nose, Mouth & $\begin{array}{c}\text { Eyebrows, } \\
\text { Eye, Nose, Mouth }\end{array}$ & $\begin{array}{c}\text { Eye, Nose, Mouth } \\
\text { Chin }\end{array}$ & $\begin{array}{c}\text { Eyebrows, Eye, } \\
\text { Nose, Mouth Chin }\end{array}$ \\
\hline $\begin{array}{c}\text { Image resolution } \\
\text { (pixels) }\end{array}$ & $28 \times 28$ & $28 \times 32$ & $28 \times 42$ & $28 \times 42$ \\
\hline
\end{tabular}


as shown in Table 1 and Fig. 3. They looked at two face images on a 19 inch liquid crystal display (LCD) monitor and determined whether two face images were from a same person. The computer program to measure human face recognition performance was implemented in Visual $\mathrm{C}^{++}$. The graphical user interface (GUI) program was displayed at the center of the screen on a gray background. To help the participants understand the recognition process and easily answer the questions during the experiment, we explained the experimental process to them before performing the human-based face recognition.

\subsubsection{Procedure}

The human-based face recognition procedure was executed as follows:

(1) The participants looked at the questions ("Same person?" or "Different person?") during the experiments which consisted of 40 pairs of face images for each case in Table 1.

(2) They determined whether or not a pair of face images was from the same person within 2 $\mathrm{s}$, as shown in Fig. 4.

(3) The accuracy of the human-based face recognition was measured for each case of Table 1 .

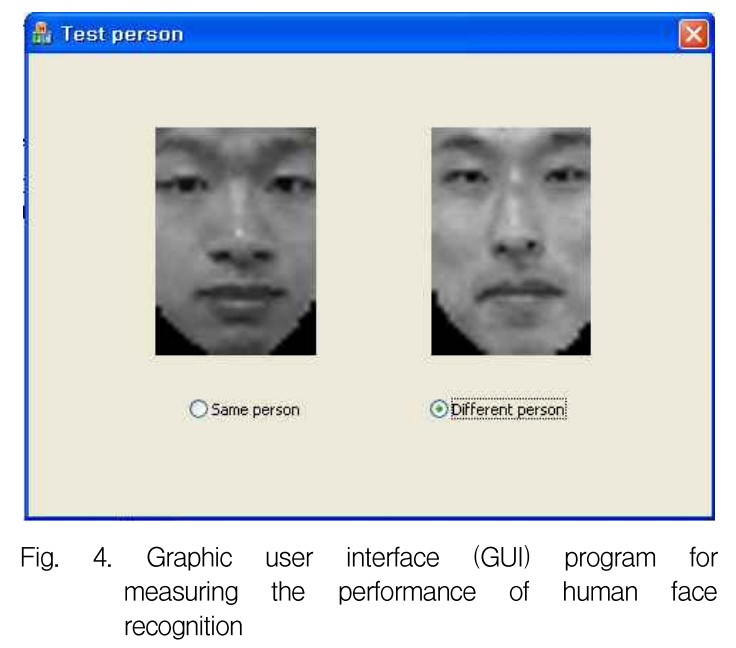

\section{EXPERIMENTAL RESULTS}

We collected 480 facial images from 48 subjects in order to test computer-based and human-based face recognition. Ten facial images per subject were captured. Since our goal is to compare the recognition performance of human and computer according to facial components, all the face data were captured without the variations of illumination, expression and pose. The size of a captured facial image was $640 \times 480$ pixels. In our computer-based face recognition, half of the facial images were used for training the PCA and the others were used for testing.

In the first experiment, we measured the accuracy of computer-based face recognition for each case in Table 1. Fig. 5 shows the receiver operating characteristic (ROC) curves which represent the genuine acceptance rate (GAR) at various false acceptance rate (FAR) levels $[40,41]$. GAR is defined as 100 - the false rejection rate (FRR) (\%). FAR means the error that occurs when an unenrolled person is accepted as enrolled, and FRR means the error that occurs when an enrolled person is rejected as unenrolled.

As shown in Fig. 5, the EER of Case 2 in Table

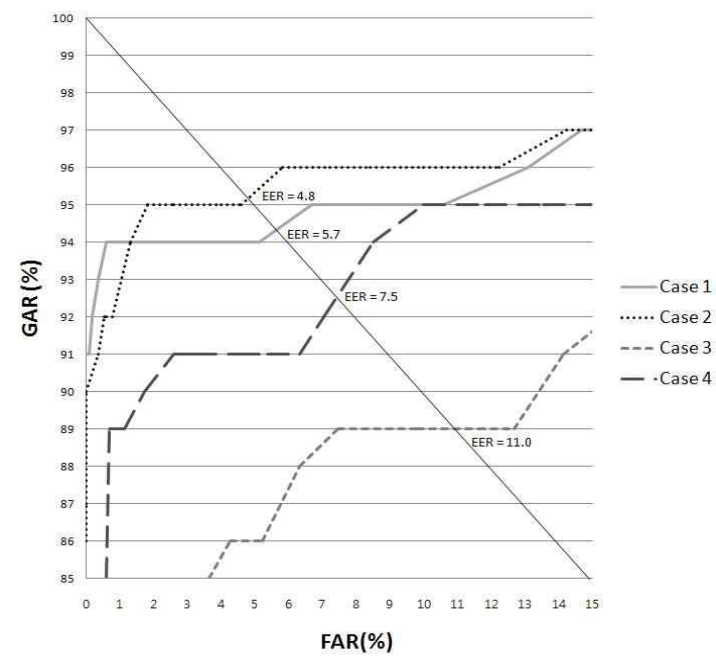

Fig. 5. ROC curves of computer-based face recognition for each case in Table 1. 
1 (including eyes, nose, mouth and eyebrows) was lower than the EER of the others. However, recognition accuracy was reduced in Case 3 and Case 4 including the chin. In general, each person had significant individual variations in chin shape and position according to the shape of the face in the captured image. These variations caused the reduction of recognition accuracy in computerbased face recognition because the alignment of each facial feature is important for high accuracy in computer-based face recognition. From the $\mathrm{ex}^{-}$ perimental results, we determined that eyebrows were an important component which enhanced the performance of face recognition, and the chin was a component which degraded the performance.

In the second experiment, we measured the accuracy of human-based face recognition for each case in Table 1. Fig. 6 shows the accuracy of human-based face recognition obtained from 20 participants. For fair comparison, the 20 participants are not familiar with the 48 subjects from which 480 facial images were collected. And the 20 participants are not experts in face recognition.

The best recognition accuracy was obtained in Case $4($ mean $=91.7 \%$, standard deviation $=0.6 \%)$ including all facial components. The performance of Case $2($ mean $=84.3 \%$, standard deviation $=0.8 \%)$ was similar to Case 3 (mean $=83.9 \%$, standard $\mathrm{de}^{-}$ viation $=0.8 \%$ ). Case 1 (mean $=77.5 \%$, standard $\mathrm{de}^{-}$ viation $=0.7 \%$ ) had the worst recognition accuracy. In the human-based face recognition, more facial information resulted in higher recognition accuracy

In the third experiment, we compared the difference between the recognition accuracies of each case in Table 1 based on t-test. As shown in Table 2 , all differences were significant excluding one between Case 2 and Case 3 .

In Table 2, the recognition accuracy of Case 4 was better than that of Case 1 as shown in Fig. 6 , with significance at the confidence level of $\mathrm{p}^{-}$value $<0.001(99.9 \%)$. The recognition accuracy

Table 2. p-values between cases in the humanbased recognition

\begin{tabular}{|c|c|c|c|}
\hline & Case 2 & Case 3 & Case 4 \\
\hline \hline Case 1 & $0.012^{*}$ & $0.014^{*}$ & $1.12 \times 10^{-7 * * *}$ \\
\hline Case 2 & - & 0.882 & $0.003^{* *}$ \\
\hline Case 3 & - & - & $0.0014^{* *}$ \\
\hline
\end{tabular}
${ }^{*}$ p-value $<0.05,{ }^{* *} \mathrm{p}^{-}$value $<0.01,{ }^{* * *} \mathrm{p}^{-}$value $<0.001$

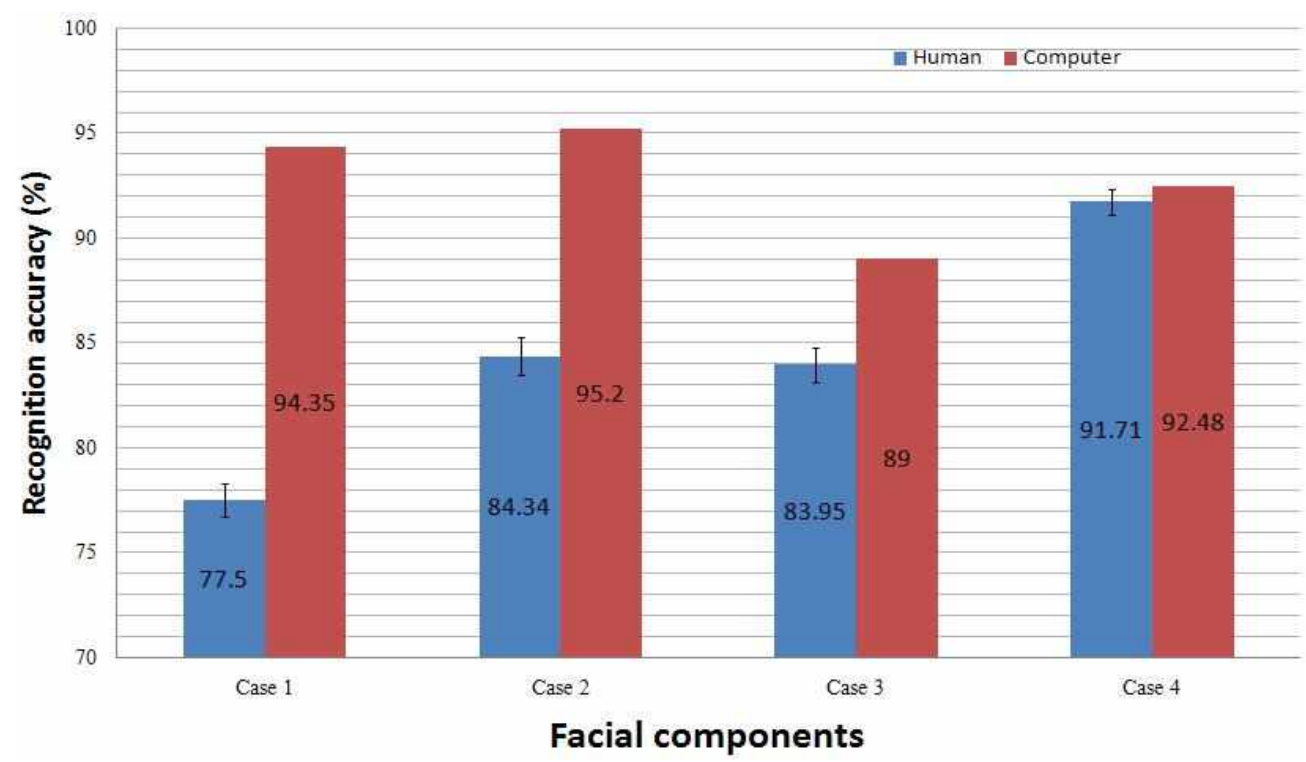

Fig 6. Accuracy levels of human-based and computer-based face recognition. 
of Case 4 was better than the accuracy levels of Cases 2 and 3 with significance at the confidence level of $\mathrm{p}^{-}$value $<0.01$ (99\%). The recognition $\mathrm{ac}^{-}$ curacy of Cases 2 and 3 were better than that of 'Case 1' with significance at the confidence level of $\mathrm{p}^{-}$-value $<0.05(95 \%)$. However, although the recognition accuracy of Case 2 was slightly better than that of Case 3 as shown in Fig. 6, the result was not significant ( $\mathrm{p}$-value of 0.882 ).

From the t-test results, we know that the face recognition accuracy of humans was enhanced when much facial information was given, and the importance of the eyebrow feature (Case 2) was similar to that of the chin feature (Case 3) from the correlation between Case 2 and Case 3. Even if the shape of the chin is changed in the captured image when performing the image- ${ }^{-}$capture proc $^{-}$ ess, recognition accuracy is not reduced because a human has the ability to transform the shape of the chin in the captured image into the shape he has remembered.

In our final experiment, we compared computer-based and human-based face recognition based on a t-test. As shown in Table 3 and Fig. 6 , the accuracy of computer-based face recognition was significantly higher than human-based face recognition in all cases excluding Case $4(\mathrm{p}$-value of 0.37). This means that the accuracy of computer-based face recognition when using a limited number of facial features such as eyes, nose, and mouth (Case 1); eyebrows, eyes, nose, and mouth (Case 2); and eyes, nose, mouth, and chin (Case 3) was better than that of human-based face

Table 3. The p-values between the accuracies of human-based and the computer-based face recognition from Fig. 6

\begin{tabular}{|c|c|}
\hline & P-value \\
\hline \hline Case 1 & $5.02 \times 10^{-12 * * *}$ \\
\hline Case 2 & $6.02 \times 10^{-7 * * *}$ \\
\hline Case 3 & $8.00 \times 10^{-3 * *}$ \\
\hline Case 4 & $3.70 \times 10^{-1}$ \\
\hline
\end{tabular}

${ }^{*} \mathrm{p}-$ value $<0.05, \quad{ }^{* *} \mathrm{p}-$ value $<0.01, \quad{ }^{* * *} \mathrm{p}-$ value $<0.001$ recognition. However, although the accuracy of computer-based recognition (about 92.5\%) was slightly better than that of human-based recognition (about 91.7\%) in Case 4, the result was not significant ( $\mathrm{p}$-value of 0.37 ).

Consistent inclusions of features such as the chin area are important for computer-based face recognition, as shown in Figs. 5 and 6. However, the number of facial features is more important for human-based face recognition.

\section{CONCLUSION}

We measured face recognition accuracy according to facial components in order to determine the effect of each component, and compared the difference between computer-based and human-based face recognition. The number of facial features (when including eyebrows, eye, nose, mouth, and chin) has a greater impact on the performance of human-based face recognition. However, consistent inclusion of features has more influence on the performance of computer-based face recognition because a computer uses the pixel values of facial images in classifying faces. In the $\mathrm{ex}^{-}$ perimental results, the eyebrow feature enhanced the accuracy of computer-based face recognition but it can be easily occluded by hair. Therefore, the occlusion problem should be solved in order to use the eyebrow feature for face recognition. In addition, we would enhance the recognition accuracy by solving the problem of inconsistent inclusion of some feature such as chin area for computer-based face recognition.

\section{ACKNOWLEDGEMENT}

This work was supported by NAP (National Agenda Project) of Korea Research Council of Fundamental Science \& Technology. 


\section{REFERENCES}

[1] G. Davies, H. Ellis, and J. Shepherd, "Cue Saliency in Faces as Assessed by the 'Photofit' Technique," Perception, Vol.6, pp. 263269, 1977.

[2] N. D. Haig, "Exploring Recognition with Interchanged Facial Features," Perception, Vol.15, pp. 505-512, 1986.

[3] I. H. Fraser, G. L. Craig, and D. M. Parker, "Reaction Time Measures of Feature Saliency in Schematic Faces," Perception, Vol.19, pp. 661-673, 1990.

[4] J. Sadrô, I. Jarudi, and P. Sinhaô, "The Role of Eyebrows in Face Recognition," Perception, Vol.32, pp. 285-293, 2003.

[5] A. J. O'Toole, P. J. Phillips, F. Jiang, J. Ayyad, N. Penard, and H. Abdi, "Face Recognition Algorithm Surpass Humans Matching Faces over Changes in Illumination," IEEE Trans. on Pattern Analysis and Machine Intelligence, Vol.29, pp. 1642-1646, 2007.

[6] R. Verma, C. Davatzikosa, J. Lougheadb, T. Indersmittenb, R. Huc, C. Kohlerb, R. E. Gurb, and R. C. Gurb, "Quantification of Facial Expressions Using High-Dimensional Shape Transformations," Journal of Neuroscience Methods, Vol.141, pp. 61-73, 2005.

[7] R. C. Gur, R. Sara, M. Hagendoorn, O. Marom, P. Hughett, L. Macy, T. Turner, R. Bajcsy, A. Posner, and R. E. Gur, "A Method for Obtaining 3-Dimensional Facial Expressions and Its Standardization for Use in Neurocognitive Studies," Journal of Neuroscience Methods, Vol.115, pp. 137-143, 2002.

[8] D.-L. Maeng, B.-W. Hong, and S.-J. Kim, "Evaluation of Face Recognition System Based on Scenarios," Journal of Korea Multimedia Society, Vol.13, No.4, pp. 487-495, 2010.

[9] A. O'Toole and M. Tistarelli, "Face Recognition in Humans and Machines," Handbook of
Remote Biometrics for Surveillance and Security, London, Springer, pp. 111-153, 2009.

[10] M. Turk and A. Pentland, "Eigenfaces for Recognition," Journal of Cognitive Neuroscience, Vol.3, pp. 71-86, 1991.

[11] M. Kirby and L. Sirovich, "Application of the Karhnen-Loeve Procedure for the Characterization of Human Faces," IEEE Trans. on Pattern Analysis and Machine Intelligence, Vol.12, pp. 103-108, 1990.

[12] R. Gottumukkal and V. K. Asari, "An Improved Face Recognition Technique Based on Modular PCA Approach," IEEE Trans. on Pattern Analysis and Machine Intelligence, Vol.28, pp. 2037-2041, 2003.

[13] H. Zhao, P. C. Yuen, and J. T. Kwok. "A Novel Incremental Principal Component Analysis and Its Application for Face Recognition," IEEE Trans. on Systems Man and Cybernetics, Part B: Cybernetics, Vol.36, pp. 873-886, 2006.

[14] H. Zou, T. Hastie, and R. Tibshirani. "Sparse Principal Component Analysis," Journal of Computational and Graphical Statistics, Vol.15, pp. 262-286, 2006.

[15] H. Zhao and P.C. Yuen "Incremental Linear Discriminant Analysis for Face Recognition," IEEE Trans. on Systems Man and Cybernetics, Part B: Cybernetics, Vol.38, pp. 210-221, 2008.

[16] J. Lu, K. N. Plataniotis, and A. N. Venetsanopoulos "Face Recognition Using LDA-based Algorithms," IEEE Trans. on Neural NetWorks, Vol.14, pp. 195-200, 2003.

[17] L. Shen, L. Bai, and F. Michael, "Gabor Wavelets and General Discriminant Analysis for Face Identification and Verification," Image and Vision Computing, Vol.25, pp. 553-563, 2007.

[18] G. Baudat and F. Anouar, "Generalized Discriminant Analysis Using A Kernel Approach," IEEE Trans. on Neural Networks, Vol.12, pp. 2385-2404, 2000. 
[19] K. R. Muller, S. Mika, G. Ratsch, K. Tsuda, and B. Scholkopf, "An Introduction to Kernel Based Learning Algorithms," IEEE Trans. on Neural Networks, Vol.12, pp.181-201, 2001.

[20] S. Mika, G. Ratsch, J. Weston, B. Scholkof, and K. R. Muller "Fisher Discriminant Analysis with Kernels," Proc. the IEEE Workshop on Neural Networks for Signal Processing, Vol.IX, pp. 41-48, 1999.

[21] L. Bao, P. Shyang, and L. Ming, "Face Recognition Using Gabor-based Complete Kernel Fisher Discriminant Analysis with Fractional Power Polynomial Models," Neural Computing and Application, Vol.18, pp.613-621, 2009.

[22] S. Mika, G. Ratsch, B. Scholkofm A. Smola, J. Weston, and K. R. Muller, "Invariant Feature Extraction and Classification in Kernel Spaces," Advanced in Neural Information Processing Systems, Vol.12, MIT Press, pp. 526-532, 2000

[23] O. Deniz, M. Castrillón, and M. Hernández, "Face Recognition Using Independent Component Analysis and Support Vector Machines," Pattern Recognition Letters, Vol.24, pp. 2153-2157, 2003.

[24] H. K. Ekenel and R. Stiefelhagen, "Analysis of Local Appearance Based Face Recognition: Effects of Feature Selection and Feature Normalization," Proc. the IEEE CVPR Biometrics Workshop, New York, USA, June 2006.

[25] F. Y. Shih and C. Chuang. "Automatic Extraction of Head and Face Boundaries and Facial Features," Information Sciences, Vol. 158, pp. 117-130, 2004.

[26] K. Sobottka and I. Pitas "A Fully Automatic Approach to Facial Feature Detection and Tracking," Proc. Audio-and Video based Biometric Person Authentication, Vol.1206, pp. 77-84, 1997.

[27] M. Zobe, A. Gebhard, D. Paulus, J. Denzler, and H. Niemann, "Robust Facial Feature
Localization by Coupled Features," Proc. the Fourth IEEE International Conference on Automatic Face and Gesture Recognition, France, pp. 2-7, 2000.

[28] L. Wiskott, J. M. Fellous, and C. V. der Malsburg, "Face Recognition by Elastic Bunch Graph Matching," IEEE Trans. on Pattern Analysis and Machine Intelligence, Vol.19, pp. 775-779. 1997.

[29] B. Abboud, F. Davoine, and M. Dang "Facial Expression Recognition and Synthesis Based on An Appearance Model," Signal Processing Image Communication, Vol.19, pp. 723-740, 2004.

[30] F. Tang and B. Deng "Facial Expression Recognition Using AAM and Local Facial Features," Proc. the third International Conference on Natural Computation, Vol.4, pp. 632-635, 2007.

[31] S. L. Gallou, G. Breton, C. Garcia, and R. Seguier, "Distance Maps: A Robust Illumination Preprocessing for Active Appearance Models," Proc. the International Conference on Computer Vision Theory and Applications, Vol.2, pp. 35-40, 2006.

[32] P. Viola and M. Jones, "Robust Real-time Face Detection," International Journal of Computer Vision, Vol.57, No.2, pp. 137-154, 2004.

[33] Open Computer Vision Library, http://opencvlibrary.sourceforge.net/(accessed November 29, 2011).

[34] Y. Cheon and D. Kim, "Natural Facial Expression Recognition Using Differential-AAM and Manifold Learning," Pattern Recognition, Vol.42, pp. 1340-1350, 2009.

[35] G. Edwards, C. Taylor, and T. Cootes, "Active Appearance Models," IEEE Trans. on Pattern Analysis and Machine Intelligence, Vol.23, pp. 681-685, 2001.

[36] I. Matthews and S. Baker, "Active Appearance Models Revisited," International Journal 
of Computer Vision, Vol.60, pp. 135-164, 2004.

[37] K. Fukunaga, Introduction to Statistical Pattern Recognition, Academic Press, New York, 1972.

[38] K. Chung, S. C. Kee, and S. R. Kim, "Face Recognition Using Principal Component Analysis of Gabor Filter Responses," Proc. International Workshop on Recognition Analysis and Tracking of Faces and Gestures in Real-Time System, pp. 53-57, 1999.

[39] Eigenface-based Facial Recognition, http:// openbio.sourceforge.net/resources/eigenfaces /eigenfaces-html/facesOptions.html (accessed on November 29, 2011).

[40] J. Wayman, "Technical Testing and Evaluation of Biometric Identification Devices," In Biometrics: Personal Identification in Networked Society, Kluwer Academic, Netherlands, pp. 345-368, 1999.

[41] A. J. Mansfield and J. L. Wayman, Best Practices in Testing and Reporting Performance of Biometric Devices, UK Government Biometrics Working Group, England, 2002.

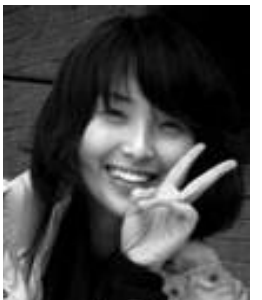

Hyun Ha Nam

2009. 2. Bachelor in Division of Digital Media Tech., Sangmyung University

2011. 2. Master in Dept. of Electronics and Electrical Eng., Dongguk University

Present: Ph.D candidate in Graduate School of Information Science and Eng., Tokyo Institute of Technology

Research interests: image signal processing, pattern recognition

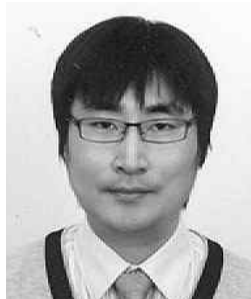

\section{Byung Jun Kang}

2004. 2. Bachelor in Dept. of Computer Science, Sangmyung University

- 2006. 2. Master in Dept. of Computer Science, Sangmyung University

2009. 2. Ph.D in Dept. of Computer Science, Sangmyung University

2009. 5. Research professor in Hanyang University

2010. 8. Senior researcher in ETRI

$\sim$ Current. Senior researcher in Hyundai Mobis

Research interests: image signal processing, pattern recognition, car vision system

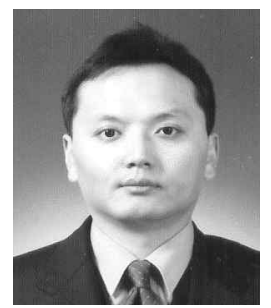

Kang Ryoung Park

1994. 2. Bachelor in Dept. of Electronics Eng., Yonsei University

1996. 2. Master in Dept. of Electronics Eng., Yonsei University

2000. 2. Ph.D in Dept. of Electrical and Computer Eng., Yonsei University

2003. 2. Senior researcher in LG Elite

- 2008. 2. Full time lecturer and assistant professor in Div. of Digital Media Tech., Sangmyung Univ

$\sim$ Current: Assistant professor and associate professor in Div. of Electronics and Electrical Eng., Dongguk University

Research interests: image signal processing, pattern recognition, biometrics 